\title{
Eletromiografia de superfície para avaliação dos músculos do assoalho pélvico feminino: revisão de literatura
}

\author{
Evaluation of female pelvic floor muscles using surface \\ electromyography: literature review
}

Ana Paula Magalhães Resende ${ }^{1}$, Mary Uchiyama Nakamura ${ }^{2}$, Elizabeth Alves Gonçalves Ferreira ${ }^{3}$, Carla Dellabarba Petricelli ${ }^{4}$ Sandra Maria Alexandre',

Míriam Raquel Diniz Zanetti ${ }^{6}$

Estudo desenvolvido no Departamento de Obstetrícia da Universidade Federa de São Paulo (UNIFESP-EPM) - São Paulo (SP), Brasil.

${ }^{1}$ Fisioterapeuta; Especialista em Fisioterapia nas Disfunções do Assoalho Pélvico e Fisioterapia em Obstetrícia pela UNIFESP-EPM; Pós-graduanda pelo Departamento de Ginecologia da UNIFESP-EPM - São Paulo (SP), Brasil.

${ }^{2}$ Médica; Professora Associada Livre Docente do Departamento de Obstetrícia da UNIFESP-EPM - São Paulo (SP), Brasil.

${ }^{3}$ Fisioterapeuta; Professora Adjunta do Curso de Fisioterapia da Universidade de São Paulo (USP) - São Paulo (SP), Brasil.

${ }^{4}$ Fisioterapeuta; Coordenadora do Curso de Especialização de Fisioterapia em Obstetrícia da UNIFESP-EPM, São Paulo (SP), Brasil; Professora do Curso de Fisioterapia da Uni Anchieta - São Paulo (SP), Brasil.

${ }^{5}$ Médica; Professora Adjunta do Departamento de Obstetrícia da UNIFESP-EPM - São Paulo (SP), Brasil.

${ }^{6}$ Fisioterapeuta; Coordenadora do Curso de Especialização de Fisioterapia em Obstetrícia da UNIFESP-EPM, cidade (SP), Brasil; Professora do Curso de Fisioterapia do centro Universitário FIEO (UNIFIEO) - Osasco (SP), Brasil

\section{ENDEREÇO PARA \\ CORRESPONDÊNCIA}

Míriam R. D. Zanetti - Rua Francisco Isoldi, 312, apto 42B - Vila Madalena - CEP: 05448-040 - São Paulo (SP),

Brasil - E-mail: mizanetti@uol.com.br

\section{APRESENTAÇÃO}

fev. 2010

ACEITO PARA PUBLICAÇÃO

set. 2010

FONTE DE FINANCIAMENTO nenhuma

CONFLITO DE INTERESSES nada a declarar
RESUM0: A eletromiografia de superfície tem grande importância clínica e de pesquisa para o fisioterapeuta. Apesar de captar a atividade elétrica promovida pelo recrutamento das unidades motoras, há boa correlação entre o número de unidades ativadas e a força muscular. É um dos métodos de maior especificidade na avaliação do assoalho pélvico, embora não haja consenso em relação à sua aplicação. Essa revisão de literatura foi desenvolvida com o objetivo de agrupar as informações sobre o uso da eletromiografia de superfície na avaliação do assoalho pélvico. Foram pesquisados artigos nas bases de dados Medline, PubMed, Lilacs, SciELO e Biblioteca Cochrane, e selecionados os que avaliassem o assoalho pélvico feminino por meio de eletromiografia de superfície. Apesar de sua metodologia ainda carecer de padronização, é um instrumento que deve ser considerado nas pesquisas científicas em nosso meio, pois parece apresentar boa reprodutibilidade e confiabilidade. Pacientes com disfunções do assoalho pélvico possuem alterações no tempo de ativação dos músculos do assoalho pélvico (MAP) e músculos abdominais. Quanto à gestação e puerpério, ainda faltam evidências sobre possíveis alterações da ativação elétrica dos MAP nesses períodos.

Descritores: soalho pélvico; eletromiografia; gravidez; incontinência urinária.

ABSTRACT: Surface electromyography has clinical and research importance for the physiotherapist. Although capturing electrical activity promoted by recruitment of motor units, there is a good correlation between the number of activated units and muscle strength. This is one of the methods of higher specificity in pelvic floor evaluation, although the lack of consensus regarding its application. The aim of this literature review was to cluster information regarding to the use of surface electromyography in the evaluation of pelvic floor. Papers were searched in Medline, Pubmed Lilacs, SciELO and Cochrane Library. Were selected papers which methods used surface electromyography to evaluate the pelvic floor. Although its methodology still lacks standardization, is an instrument that should be considered in scientific research in our country because it seems to have good reproducibility and reliability. Women with pelvic floor disorders have changes in the activation time of the pelvic floor muscles (PFM) and abdominal muscles. With respect to pregnancy and postpartum, there is a lack of evidence on possible changes in electrical activation of PFM in these periods.

KeYwords: pelvic floor; electromyography; pregnancy; urinary incontinence. 


\section{INTRODUCÃO}

Arnold Kegel, o primeiro a descrever os exercícios para os músculos do assoalho pélvico (MAP), foi pioneiro na avaliação desses músculos por meio de um instrumento denominado perineômetro ${ }^{1}$. Embora seja amplamente utilizado na prática clínica atual, tem sua eficácia questionada no que diz respeito à avaliação, pois registra também o aumento de pressão abdominal durante as contrações dos MAP. Em busca de avaliações mais precisas da função muscular do assoalho pélvico, novos métodos foram desenvolvidos e dentre eles destaca-se a eletromiografia de superfície (EMGs).

Eletromiografia é definida como o registro extracelular da atividade bioelétrica gerada pelas fibras musculares ${ }^{2}$. Pode ser realizada por meio de agulha, que capta a atividade elétrica de poucas unidades motoras; ou por meio de eletrodo de superfície, que mensura a atividade elétrica de várias unidades motoras ao mesmo tempo ${ }^{2}$. Apesar de captar a atividade elétrica promovida pelo recrutamento das unidades motoras e não a força muscular, Vodusek ${ }^{3}$ afirma que há boa correlação entre o número de unidades motoras ativadas e a força muscular.

Atualmente, observa-se o seu uso em diversos estudos sobre avaliação do assoalho pélvico, envolvendo mulheres com e sem disfunções dessa musculatura ${ }^{4-6}$. Alguns trabalhos que utilizaram a EMGs do assoalho pélvico constataram o sinergismo da musculatura abdominal, de membros superiores e antigravitacionais bem como o tempo de ativação do assoalho pélvico e dos músculos sinérgicos pode modificar-se de acordo com a situação do assoalho pélvico e a fase de vida da mulher ${ }^{7,8}$.

Esse instrumento tem sido cada vez mais usado por fisioterapeutas tanto no atendimento clínico quanto em pesquisas científicas, mas ainda falta consenso sobre vários aspectos do método. Posicionamento do sensor e do paciente, número de contrações de fibras fásicas, tempo de contração de fibras tônicas, necessidade de avaliação concomitante de musculatura sinergista e possibilidade de utilização em situações especiais ainda devem ser padronizados.

Por isso, essa revisão foi desenvolvida com o objetivo de agrupar as informações relacionadas ao uso da eletromiografia de superfície na avaliação do assoalho pélvico.

\section{METODOLOGIA}

Após busca nos bancos de dados Medline, PubMed, Lilacs, SciELO e Biblioteca Cochrane, foram encontrados artigos publicados entre os anos de 1988 a 2010. Utilizaram-se para a pesquisa os seguintes descritores: eletromiografia, atividade elétrica, músculos do assoalho pélvico, eletromiografia de superfície; electromyography, electrical activity, pelvic floor muscles e surface electromyography; e incluídos artigos nas línguas portuguesa e inglesa.

Inicialmente foram encontrados 247 artigos sobre avaliação do assoalho pélvico, sendo 118 excluídos após a leitura do título e abstract por não utilizarem EMGs, 58 por não avaliarem o assoalho pélvico feminino e 61 porque utilizaram o método como biofeedback para tratar o assoalho pélvico de mulheres com disfunções coloproctológicas. Ao final, após excluir os artigos duplicados nas bases de dados Medline e Pubmed, 14 foram selecionados, analisados e distribuídos em categorias.

\section{RESULTADOS E DISCUSSÃO}

Foram encontrados estudos observacionais, que avaliaram a atividade elétrica dos músculos do assoalho pélvico de determinada população e compararam seus resultados nas diversas posições e com outros grupos.

Dentre os métodos de avaliação utilizados, destaca-se a associação da EMGs à perineometria e à palpação bidigital do assoalho pélvico em diversos estudos. No que se refere à avaliação de fibras rápidas e lentas e posicionamento do eletrodo, não foram encontrados dados detalhados nos estudos.

Observou-se que é baixo o número de pacientes que compõe as amostras e que há muita diversidade na mesma: mulheres nuligestas, com partos vaginais anteriores, incontinentes, continentes, pós-menopausadas, gestantes, puérperas.

\section{Eletromiografia de superfície dos musculos do assoalho pélvico e avaliação de fibras \\ fásicas e tônicas}

Joseph Santiesteban foi um dos primeiros a descrever, em 1988, sobre eletromiografia de superfície para avaliação da atividade elétrica dos MAP. Avaliou 15 mulheres (10 nulíparas e 5 gestantes) por meio de perineometria e EMGs com sensor intravaginal e comparou os resultados dos dois grupos. As mensurações foram feitas nas posições supina, decúbito lateral, ponte associada à retroversão pélvica e em pé. As contrações eram mantidas por 3, 10, 30 e 60 segundos, com eletrodos de superfície colocados nos abdutores, adutores e extensores de quadril, e músculos abdominais inferiores, além do eletrodo de superfície intravaginal. Apesar do número reduzido de pacientes, seus resultados não mostraram diferenças significativas na atividade elétrica e força muscular entre os grupos estudados ${ }^{9}$.

O tipo de eletrodo utilizado e os parâmetros de análise são fatores importantes para a discussão da eletromiografia de superfície. Para Deffieux et al. ${ }^{10}$, eletrodos de superfície são mais adequados para a captação da atividade elétrica dos MAP porque se acoplam e captam sinal melhor que os eletrodos de agulha, que são mais dolorosos e podem se deslocar durante o movimento provocado pela contração muscular.

Uma das principais variáveis analisadas com a eletromiografia de superfície é a contração voluntária máxima (CVM), realizada pelas fibras musculares rápidas, tipo II, responsáveis pela potência muscular ${ }^{4}$. Para isso, solicita-se mais de uma contração dos MAP e registra a maior de todas, pois a primeira pode ser considerada parte do aprendizado ${ }^{11}$. Grande parte dos estudos utiliza três contrações com 20 a 30 segundos de repouso entre elas ${ }^{4,5}$. Alguns autores levam em conta o valor da média de duas ou três contrações ${ }^{12}$. Por ser musculatura de alta fadigabilidade, acreditamos que as três contrações são suficientes e que provavelmente a utilização da melhor contração seja o parâmetro mais fidedigno. Se a primeira deve ser considerada um aprendizado, o cálculo da média não seria fidedigno ${ }^{11}$. 
Quanto à avaliação do endurance muscular, alguns autores utilizam a análise espectral do gráfico de EMGs e outros, o tempo de manutenção da contração em segundos ${ }^{5,13}$. A mensuração do endurance muscular visa a avaliação das fibras musculares tipo I, de contração lenta. Entretanto, é preciso cautela para analisar o tempo de contração em segundos, pois algumas pesquisas que verificaram fadiga muscular por meio de eletromiografia mostraram que os esfíncteres anal e uretral entram em fadiga após um minuto de contração voluntária sustentada. Observou-se aumento no recrutamento de unidades motoras durante contrações máximas repetidas ou sustentadas submáximas para manter o nível de força requerido ${ }^{14}$.

Resta ainda definir como o tempo de manutenção deve ser considerado, já que a contração, mesmo sustentada, não apresenta sinal mioelétrico constante, mas pequenas oscilações. Dessa forma, a análise espectral do gráfico, que envolve o começo, meio e fim da contração, parece ser mais adequada.

Algumas pesquisas associaram a EMGs com outras técnicas de avaliação do assoalho pélvico, como perineometria e palpação bidigital. Entretanto, ainda não há evidências de que estes métodos possam ser comparados entre si ${ }^{15,16}$.

\section{Sinergismo dos MAP com outros grupos musculares}

A EMGs foi utilizada para mensurar a atividade elétrica dos MAP em diversas posturas corporais e durante a contração de grupos musculares específicos ${ }^{6,8,15}$.

A atividade biomecânica dos músculos do assoalho pélvico mostrou-se bastante complexa, pois em muitos movimentos age sinergicamente devido às suas comunicações pelas fáscias musculares. É o caso do músculo transverso do abdome, que se comunica com o assoalho pélvico por meio de sua fáscia e que, quando solicitado, realiza estabilização do tronco em movimentos diversos e recruta os MAP para auxiliar na manutenção desta postura ${ }^{7,8}$.

Ao avaliar a ativação elétrica dos MAP durante movimentação dos membros superiores, que causaram desestabilização da coluna, Hodges et al. ${ }^{8}$ mostraram que há aumento da resposta dos MAP durante a contração do músculo deltóide como parte de um ajuste postural antecipatório. Esta atividade foi indiferente com relação à direção do movimento do braço. Eles ainda realizaram registros da atividade dos MAP durante algumas manobras respiratórias como respiração tranquila, respiração mantida e forçada. Observaram que os MAP foram ativados na fase expiratória ${ }^{8}$.

Sapsford et al. ${ }^{7}$ avaliaram a atividade de repouso dos MAP e dos músculos abdominais durante diferentes posições sentadas. A amostra foi composta por 17 mulheres com pelo menos um parto vaginal anterior. Os resultados mostraram que a atividade dos MAP é significativamente maior na postura sentada sem apoio e com a coluna ereta, ou seja, com ativação dos músculos paravertebrais, em comparação com a postura sentada com apoio $(p<0,01)$. Os autores sugerem que a postura corporal é importante durante a reabilitação dos MAP.

$\mathrm{Na}$ tentativa de descrever a sinergia dos MAP com a musculatura abdominal, Madill e McLean ${ }^{6}$ avaliaram a CVM de 15 nulíparas nas posições sentada, decúbito dorsal e ortostática. Avaliaram ainda a ativação dos músculos retos abdominais, oblíquos internos e externos e transversos abdominais por meio de eletrodos de superfície. Concluíram que a atividade elétrica dos MAP durante contração voluntária nas diferentes posições foi semelhante, entretanto, a sequência de ativação muscular foi diferente nas diversas posições. Na deitada, o músculo oblíquo externo é ativado antes dos outros ( $p=0,043)$, já na posição ortostática, os músculos retos abdominais e oblíquos externos são ativados antes dos MAP e os transverso abdominal e oblíquo interno são ativados depois dos MAP $(p<0,001)$. Segundo as autoras, esses resultados podem ser úteis no desenvolvimento de programas de treinamento, visando à combinação apropriada e o tempo de ativação correto para produzir fechamento efetivo da uretra.

Em discordância com este estudo estão os dados de uma pesquisa brasileira realizada por Rett et al. ${ }^{17}$, que avaliaram a contratilidade dos MAP nas posições de decúbito dorsal, sentada e ortostática. Os autores analisaram 25 mulheres que já haviam sido submetidas a tratamento para incontinência urinária de esforço (IUE). As mensurações foram obtidas por meio de probe intravaginal e o protocolo de avaliação foi um minuto de repouso para avaliar o tônus basal, 5 contrações fásicas e duas tônicas, sendo uma de 10 segundos e outra de 20 segundos. Os resultados mostraram que a atividade elétrica em decúbito dorsal foi significativamente maior que na posição ortostática nas mensurações fásicas $(p<0,01)$, tônicas de 10 segundos $(p<0,01)$ e tônicas de 20 segundos $(p=0,03)$, assim como na avaliação do tônus de base $(p=0,01)$. Os autores sugerem que o fortalecimento muscular deve ser intensificado na posição ortostática, já que as mulheres apresentaram menor atividade elétrica durante a contração ativa nesta posição.

Ainda com relação ao sinergismo muscular, alguns autores preconizam que a contração ativa de outros músculos associada à contração dos MAP aumenta sua atividade elétrica. É o caso dos músculos glúteos e adutores. Peschers et al. ${ }^{15}$ avaliaram os MAP em contração isolada e associada à contração dos glúteos, adutores e à manobra de Valsalva. Observaram que quando a contração dos MAP é associada à contração dos glúteos e dos adutores, a atividade elétrica é maior em comparação com a contração isolada $(p<0,05)$.

Junginger et al. ${ }^{18}$ avaliaram a posição do colo vesical e o aumento de pressão abdominal durante contrações dos MAP e do músculo transverso do abdome monitoradas por EMGs. As mudanças no colo vesical foram observadas por meio de ultrassonografia perineal e a pressão intra-abdominal foi monitorada com balão retal. Nove mulheres sem disfunções dos MAP foram avaliadas e realizaram contrações discretas, moderadas e máximas dos MAP e transverso do abdome, bem como manobras de Valsalva e movimento de levantar a cabeça. O colo vesical foi elevado de 1 a 3,3 mm durante as contrações discreta e moderada dos MAP e do transverso do abdome ( $p<0,0001)$. A elevação do colo vesical durante a contração discreta dos MAP foi mais significante do que todos os outros testes $(p<0,0400)$. A pressão intraabdominal aumentou para $0,26 \mathrm{cmH}_{2} \mathrm{O}$ na contração do músculo transverso do 
abdome e para $1,59 \mathrm{cmH}_{2} \mathrm{O}$ na manobra de Valsalva. A contração máxima dos MAP associada à contração abdominal aumentou a pressão intra-abdominal. Foi sugerido que os MAP devem ser treinados junto com o músculo transverso do abdome ${ }^{18}$.

Contudo, em revisão sistemática recente, Bo et al. ${ }^{11}$ afirmam que não existem evidências científicas suficientes para associar o treinamento dos MAP à contração do músculo transverso abdominal.

Alguns pontos podem ser ressaltados para a prática clínica ao utilizar a EMGs como método de avaliação do assoalho pélvico como: necessidade de se monitorar a musculatura abdominal, padronizar o posicionamento da pelve e de membros superiores e realizar a coleta do sinal sempre no mesmo momento respiratório em todas as pacientes.

\section{EMGs durante a gestação e puerpério}

Alguns períodos do ciclo vital feminino instigam uma série de especulações com relação à segurança e viabilidade de procedimentos fisioterapêuticos. Por isso, consideramos de extrema importância a abordagem da avaliação eletromiográfica do assoalho pélvico na gestação e puerpério.

Pereira et al. ${ }^{12}$ avaliaram 75 primíparas no terceiro trimestre de gestação e compararam com nova avaliação 40 dias após o parto. As voluntárias foram divididas em três grupos, de acordo com o tipo de parto: vaginal, cesariana eletiva e cesariana em vigência de trabalho de parto. O valor médio da contração voluntária máxima das gestantes foi de 35,5 $\mu \mathrm{v}$. No período puerperal, os valores encontrados foram: 31,1 $\mu \mathrm{v}$ para o grupo que se submeteu ao parto vaginal, $38,5 \mu \mathrm{v}$ para o grupo de cesariana eletiva e 32,6 $\mu \mathrm{v}$ para o grupo de cesariana em vigência de trabalho de parto. A autora concluiu que o parto vaginal provoca diminuição da contratilidade do assoalho pélvico 45 dias após o parto.

O efeito do parto vaginal na integridade dos MAP de primíparas foi comparado com atividade elétrica dos MAP de nulíparas $^{16}$. O primeiro grupo era composto por dez nulíparas e o segundo por dez primíparas com nove a dez meses de pós-parto vaginal. As mensurações foram feitas posicionando a parte metálica do probe em contato com as regiões anterior e posterior do assoalho pélvico. Os valores encontrados para o grupo de nulíparas foram significativamente maiores na CVM anterior $(p=0,001)$ e posterior $(p=0,044)$ quando comparados com as primíparas ${ }^{16}$.

Até o momento, existem poucas informações consistentes sobre as alterações da atividade elétrica do assoalho pélvico durante a gestação e no puerpério. Seria de grande relevância investigar a repercussão do parto na atividade elétrica dos MAP, correlacionando com a presença de episiotomia, o peso do feto ao nascer e o ganho de peso durante a gestação, fatores considerados de risco para o desenvolvimento de afecções do assoalho pélvico.

\section{EMGs e disfunções do assoalho pélvico}

No que tange os efeitos da idade na atividade elétrica dos MAP, Aukee et al. ${ }^{19}$ observaram menor atividade elétrica dos MAP quanto maior a idade das mulheres, mesmo nas continentes, correlacionando a diminuição da atividade elétrica dos MAP ao envelhecimento.

Foi comprovado ainda que mulheres com disfunções possuem fraqueza dos MAP e dissinergia entre a contração dos músculos abdominais e dos MAP, com tempo diferente de ativação destes músculos durante os aumentos de pressão intra-abdominal. Nas mulheres incontinentes, os músculos oblíquo interno e oblíquo externo são ativados antes dos MAP20.

Ao redor da cavidade abdominopélvica, os músculos formam um cilindro flexível que responde rapidamente às alterações de pressão intra-abdominal durante a ativação dos músculos do tronco e mudanças posturais ${ }^{12}$. Mulheres com IUE possuem aumento da atividade elétrica da musculatura do tronco que está diretamente associado a alterações no controle postural, inclusive com modificações no centro de gravidade ${ }^{21}$.

Ressalta-se ainda que a IUE é uma das principais causas de disfunção do assoalho pélvico feminino e, talvez por isso, a maioria dos artigos encontrados são direcionados a este tema.

DeLancey et al. ${ }^{20}$ descreveram diferenças na inervação da musculatura profunda e superficial do assoalho pélvi$\mathrm{co}$, indicando diferentes funções para os dois grupos musculares. Devreese et al. ${ }^{22}$ avaliaram 32 mulheres saudáveis, sem disfunções do assoalho pélvico e 50 muIheres com IUE. Utilizaram EMGs intravaginal e perineal por meio de eletrodos na musculatura superficial do assoalho pélvico em seis posições diferentes. Verificaram que as mulheres saudáveis contraem primeiramente os músculos superficiais, e logo em seguida os músculos profundos do assoalho pélvico. $\mathrm{O}$ inverso ocorreu em mulheres com IUE em três das seis posições estudadas, ou seja, a sequência de ativação muscular é inversa. Concluíram que este fator pode ser determinante para a continência e que é aconselhável considerar essas diferenciações musculares no tratamento da IUE.

Madill et al. ${ }^{14}$ concluíram que a sequência de ativação dos músculos abdominais e MAP é diferente em mulheres incontinentes quando comparadas às continentes, e que nas mulheres incontinentes os músculos abdominais são ativados antes dos MAP. O mecanismo de ativação destes músculos precisa ser melhor compreendido porque informações advindas de estudos nesta área podem colaborar de forma consistente para políticas de prevenção e tratamento da incontinência urinária.

Em acordo com esses dados está a pesquisa de Deffieux et al. ${ }^{10}$, que avaliaram a resposta do esfíncter anal externo e fibras distais do músculo levantador do ânus durante a manobra de Valsalva por meio de EMGs em 21 mulheres com incontinência urinária. A resposta adequada seria aumentar a contração dos MAP proporcionalmente ao incremento de pressão abdominal para haver fechamento uretral sem perda urinária, porém isso não aconteceu. Os autores sugerem diminuição da modulação muscular em resposta aos aumentos de pressão abdominal nessas mulheres.

Ao realizar avaliação com EMGs de pacientes que apresentem essas disfunções de assoalho pélvico, os estudos apresentados devem ser considerados. 
Mostram que serão encontradas respostas elétricas diferentes entre mulheres com e sem incontinência urinária, que a resposta sinérgica de musculatura abdominal e de tronco é alterada e que a resposta da MAP frente a aumento de pressão intraabdominal é afetada ${ }^{13}$.

\section{Validade e reprodutibilidade do método}

No que se refere à validade dos sinais da EMGs durante a contração dos MAP, alguns autores descrevem que a discriminação exata da origem da atividade elétrica ainda não é possível ${ }^{2,3,15}$, ou seja, pode ser que haja interferência de outros músculos durante a captação, denominada cross-talk ${ }^{5}$.

Para tanto, foram sugeridos alguns cuidados que, durante a mensuração, diminuiriam consideravelmente a possibilidade de captação de atividade elétrica dos músculos vizinhos. Inicialmente deve-se verificar se a paciente consegue contrair os MAP isoladamente, de forma que não haja contração visível ou palpável de outros grupos musculares como os glúteos adutores e abdominais. Sugere-se ainda observar o movimento de fechamento da vagina associado à elevação cranial do probe $^{11,15}$.
Com o objetivo de avaliar a reprodutilibidade interobservador da técnica, Grape et al. ${ }^{5}$ realizaram EMGs em 17 nulíparas que possuíam habilidade de contrair adequadamente os MAP5 . As voluntárias foram posicionadas em decúbito dorsal, com os joelhos flexionados. Foram realizadas duas mensurações com intervalos de 30 minutos entre elas e uma última mensuração de 26 a 30 dias depois. Em cada sessão foi solicitado à voluntária que realizasse três contrações máximas, dez segundos de contração mantida e dez segundos de repouso. Todas as mensurações demonstraram ter boa e alta reprodutibilidade (ICC $=0,83$ a 0,96). Quando correlacionados os melhores valores das três contrações máximas, o nível de concordância foi ainda maior do que quando correlacionadas as médias das três contrações. Portanto, a EMGs parece ser um método reprodutível para avaliação da atividade elétrica dos MAP.

Auchincloss e McLean ${ }^{4}$ testaram a reprodutibilidade interobservador da eletromiografia de superfície em dez nuligestas sem histórico de disfunções do assoalho pélvico. Encontraram uma correlação boa e alta em dois tipos de probe testados (ICC $=0,58-0,98$ e 0,80-0,98).
Não foram encontrados estudos de EMGs sobre a reprodutibilidade intra e interavaliador no período gravídicopuerperal em mulheres com ou sem disfunção do assoalho pélvico, o que retrata a carência de estudos especificamente nesta população.

\section{CONSIDERAÇÕES FINAIS}

Por ser a EMGs um método de boa aceitação por parte das pacientes e de grande importância clínica e de pesquisa, tende a ser empregado com maior frequência na área da fisioterapia. Apesar de alguns estudos mostrarem boa reprodutibilidade e confiabilidade ${ }^{4,5}$, carece ainda de padronizações com relação à metodologia a ser empregada na avaliação do assoalho pélvico. No que se refere às disfunções dessa musculatura, pacientes com incontinência urinária de esforço possuem alterações na ativação dos MAP e músculos abdominais durante as manobras de Valsalva, sugerindo deficiência de ativação muscular nas diferentes atividades. Quanto à gestação e puerpério, ainda faltam evidências sobre possíveis alterações da ativação elétrica dos MAP nesses períodos.

\section{REFERÊNCIAS}

1. Kegel AH. Progressive resistance exercise in the functional restoration of the perineal muscles. Am J Obstet Gynecol. 1948;56(2):238-48.

2. Vodusek DB. Electromyography In: Evidence based physical therapy for the pelvic floor. Elsevier, 2007, pg. 53-63.

3. Vodusek DB. The role of electrophysiology in the evaluation of incontinence and prolapse. Curr Opin Obstet Gynecol. 2002;14(5):509-14.

4. Auchincloss CC, McLean L. The reliability of surface EMG recorded from the pelvic floor muscles. J Neurosci Methods; 2009;182(1):85-96.

5. Grape HH, Dedering A, Jonasson AF. Retest reliability of surface electromyography on the pelvic floor muscles. Neurourol Urodyn. 2009;28(5):395-9.

6. Madill SJ, McLean L. Quantification of abdominal and pelvic floor muscle synergies in response to voluntary pelvic floor muscle contractions. J Electromyogr Kinesiol. 2008;18(6):955-64.
7. Sapsford RR, Richardson CA, Maher CF, Hodges PW. Pelvic floor muscle activity in different sitting postures in continent and incontinent women. Arch Phys Med Rehabil. 2008;89(9):1741-7.

8. Hodges PW, Sapsford R, Pengel LHM. Postural and respiratory function of the pelvic floor muscles. Neurourol Urodyn. 2007;26:362-71.

9. Santiesteban AJ. Electromyographic and dynamometric characteristics of female pelvic-floor musculature. Phys Ther. 1988;68(3):344-51.

10. Deffieux X, Hubeaux K, Porcher R, Ismael SS, Raibaut $P$, Amarenco G. Pelvic floor muscle activity during coughing: altered pattern in women with stress urinary incontinence. Urology. 2007;70(3):443-7.

11. Bo K, Kvarstein B, Hagen RR, Larsen S. Pelvic floor muscle exercise for the treatment of female stress urinary incontinence: II. Validity of vaginal pressure measurements of pelvic floor muscle strength and the necessity of supplementary methods for control of correct contraction. Neurourol Urodyn. 1990;9(5):479-87. 


\section{Referências (cont.)}

12. Botelho S, Riccetto C, Herrmann V, Pereira LC, Amorim C, Palma P. Impact $f$ delivery mode on electromyographic activity of pelvic floor: comparative prospective study. Neurourology and Urodynamics. 2010;29(7):1258-61.

13. Bo K, Morkved S, Frawley H, Sherburn M. Evidence for benefit of transversus abdominis training alone or in combination with pelvic floor muscle training to treat female urinary incontinence: a systematic review. Neurourol Urodyn. 2009;28(5):368-73.

14. Madill SJ, Harvey MA, McLean L. Women with SUI demonstrate motor control differences during voluntary pelvic floor muscle contractions. Int Urogynecol J Pelvic Floor Dysfunct. 2009;20(4):447-59.

15. Peschers UM, Gingelmaier A, Jundt K, Leib B, Dimpfl T. Evaluation of pelvic floor muscle strength using four different techniques. Int Urogynecol J Pelvic Floor Dysfunct. 2001;12(1):27-30.

16. Marshall K, Walsh DM, Baxter GD. The effect of a first vaginal delivery on the integrity of the pelvic floor musculature. Clin Rehabil. 2002;16(7):795-9.

17. Rett MT, Simões JA, Herrmann V, Marques AA, Morais SS. Existe diferença na contratilidade da musculatura do assoalho pélvico feminino em diversas posições? Rev Bras Ginecol Obstet. 2005;27(1):20-3.

18.Junginger B, Baessler K, Sapsford R, Hodges PW. Effect of abdominal and pelvic floor tasks on muscle activity, abdominal pressure and bladder neck. Int Urogynecol J. 2010;21(1):69-77.

19.Aukee P, Penttinen J, Airaksinen O. The effect of aging on electromyographic activity of pelvic floor muscles. A comparative study among stress incontinent patients and asymptomatic women. Maturitas. 2003;44(4):253-7.

20.DeLancey JO. Anterior pelvic floor anatomy in female. In: The pelvic floor: Its function and disorders, 2 ed. New York: Harcourt and Elsevier, 2002, pg 13-28.

21.Smith MD, Coppieters MW, Hodges PW. Is balance different in women with and without stress urinary incontinence? Neurourol Urodyn. 2008;27(1):71-8.

22.Devreese A, Staes F, Janssens L, Penninckx F, Vereecken R, De Weerdt W. Incontinent women have altered pelvic floor muscle contraction patterns. J Urol. 2007;178(2):558-62. 